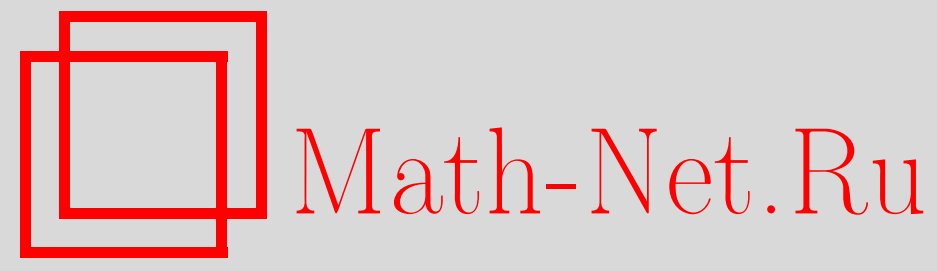

Х. Вальтер, А. Л. Скубачевский, О гиперболичности быстро осциллирующих периодических решений функционально-дифференциальных уравнений, Функи. анализ и его прил., 2005, том 39, выпуск $1,82-85$

DOI: https://doi.org/10.4213/faa33

Использование Общероссийского математического портала MathNet.Ru подразумевает, что вы прочитали и согласны с пользовательским соглашением

http://www. mathnet.ru/rus/agreement

Параметры загрузки:

IP : 54.197 .217 .227

26 апреля 2023 г., 11:39:59

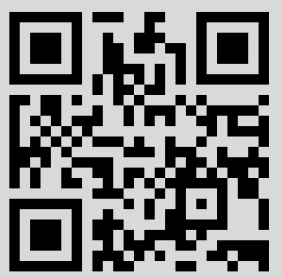




\title{
О гиперболичности быстро осциллирующих периодических решений функционально-дифференциальных уравнений
}

\author{
(c) 2005. Х.-О. ВАЛЬТЕР, А. Л. СКУБАЧЕВСКИЙ
}

1. Рассмотрим периодические решения уравнения

$$
x^{\prime}(t)=-\mu x(t)+f(x(t-1)),
$$

где $\mu>0$ и $f: \mathbb{R} \rightarrow \mathbb{R}$ - нечетная непрерывно дифференцируемая функция, такая, что $f(y)>0$ при $y>0$. В случае рациональных периодов, меньших чем 2 , мы получим критерий гиперболичности периодического решения $x$. В случае рациональных периодов, больших чем 2 , критерий гиперболичности периодических решений был получен в [1].

2. Пусть $C=C([-1,0], \mathbb{R})$ и $C_{\mathbb{C}}=C([-1,0], \mathbb{C})$ - банаховы пространства с нормой $\|\varphi\|=\max _{t \in[-1,0]}|\varphi(t)|$. Под решением уравнения (1) понимается либо вещественнозначная дифференцируемая на $\mathbb{R}$ функция, удовлетворяющая уравнению (1) всюду, либо вещественнозначная непрерывная функция, определенная на некотором неограниченном полуинтервале $\left[t_{0}-1, \infty\right), t_{0} \in \mathbb{R}$, которая дифференцируема и удовлетворяет уравнению (1) для любого $t>t_{0}$. Если $y$ - отображение интервала $[t-1, t]$ в множество $B$, то функция $y_{t}:[-1,0] \rightarrow B$ определяется по формуле $y_{t}(s)=y(t+s)$. Таким образом, решения $x=y: \mathbb{R} \rightarrow \mathbb{R}$ уравнения (1) определяют орбиты $o(y)=\left\{y_{t}: t \in \mathbb{R}\right\} \subset C$.

Предположим, что существует $2 r$-периодическое решение $x: \mathbb{R} \rightarrow \mathbb{R}$ уравнения (1), имеющее симметрию $x(t)=-x(t+r)$ для всех $t \in \mathbb{R}$. Поведение орбит $o(y)$ решений уравнения (1), близких к периодической орбите $o(x)=\left\{x_{t}: t \in \mathbb{R}\right\}$, определяется спектром оператора монодромии $\mathscr{M}: C_{\mathbb{C}} \rightarrow C_{\mathbb{C}}$, заданного формулой $\mathscr{M} \varphi=v_{2 r}^{\varphi}$, где $v^{\varphi}:[-1, \infty) \rightarrow \mathbb{C}$ - решение начальной задачи

$$
\begin{aligned}
v^{\prime}(t) & =-\mu v(t)+f^{\prime}(x(t-1)) v(t-1) & & (t>0), \\
v(t) & =\varphi(t) & & (t \in[-1,0]) .
\end{aligned}
$$

Оператор $\mathscr{M}^{j}$, где $j \in \mathbb{N}$ и $j 2 r \geqslant 1$, является компактным. Поэтому все точки спектра $\lambda \neq 0$ оператора $\mathscr{M}$ - изолированные собственные значения конечной алгебраической кратности $m(\mathscr{M}, \lambda)$. Эти собственные значения называются мультипликаторами Флоке. Число 1 является мультипликатором Флоке, соответствующим собственному вектору $x_{0}^{\prime}$. Результаты о мультипликаторах Флоке для периодических решений различных функционально-дифференциальных уравнений см. в [1-7]. Если мультипликатор Флоке 1 простой $(m(\mathscr{M}, 1)=1)$ и единичная окружность не содержит других мультипликаторов Флоке, то периодическое решение $x$ называется гиперболическим. В этом случае все орбиты,

* Работа второго автора выполнена при финансовой поддержке Mercator programm der Deutschen Forschungsgemeinschaft, РФФИ (грант №04-01-00256) и Министерства образования и науки РФ (грант №E02-1.0-131). 
близкие к $o(x)$ и определенные на неограниченных полуинтервалах, сходятся экспоненциально к $o(x)$ при $|t| \rightarrow \infty$, см. гл. 10 в [2] и гл. XIV в [6].

3. Мы введем оператор $V: C_{\mathbb{C}} \rightarrow C_{\mathbb{C}}$ по формуле $(V \varphi)(t)=v^{\varphi}(r+t)$ для всех $\varphi \in C_{\mathbb{C}}, t \in[-1,0]$, где $v^{\varphi}:[-1, \infty) \rightarrow \mathbb{C}$ - решение начальной задачи $(2),(3)$. Очевидно, что $\mathscr{M}=V^{2}$ и -1 является собственным значением оператора $V$.

Установим взаимосвязь между уравнением

$$
(V-\lambda I) \varphi=\psi
$$

и краевой задачей для системы обыкновенных дифференциальных уравнений, где $\lambda \neq 0$ и $\psi \in C_{\mathbb{C}}$ заданы.

Пусть $r=N / M$, где целые числа $M>N>0$ взаимно простые. Положим $m=\min \{s \in \mathbb{N}: s N>M\}$. Так как $N<M$, то $m \geqslant 2$. Пусть $n=M-(m-1) N$. Очевидно, что $0<n<N$. Положим $\tau=1 / M$. Тогда $r=N \tau$ и $1=M \tau$. Введем вектор-функцию $U:[0, \tau] \rightarrow \mathbb{C}^{N}$ с координатами $u_{1}, \ldots, u_{N}$ по формуле

$$
u_{i}(t)=v^{\varphi}(t+(i-1) \tau) \quad(t \in[0, \tau], i=1, \ldots, N) .
$$

Рассмотрим систему обыкновенных дифференциальных уравнений

$$
\begin{array}{r}
u_{i}^{\prime}(t)=-\mu u_{i}(t)+\lambda^{-m} a_{i}(t) u_{i+N-n}(t)-a_{i}(t) \sum_{s=1}^{m} \lambda^{-s} \psi_{i+(s-1) N}(t) \\
(t \in(0, \tau), i=1, \ldots, n), \\
u_{i}^{\prime}(t)=-\mu u_{i}(t)+\lambda^{-(m-1)} a_{i}(t) u_{i-n}(t)-a_{i}(t) \sum_{s=1}^{m-1} \lambda^{-s} \psi_{i+(s-1) N}(t) \\
(t \in(0, \tau), i=n+1, \ldots, N)
\end{array}
$$

с краевыми условиями

$$
u_{i+1}(0)-u_{i}(\tau)=0 \quad(i=1, \ldots, N-1), \quad u_{1}(0)-\lambda^{-1} u_{N}(\tau)=-\lambda^{-1} \psi_{M}(\tau),
$$

где $a_{i}(t)=f^{\prime}(x(t+(i-1-M) \tau))(t \in[0, \tau], i=1, \ldots, N), \psi_{j}(t)=\psi(t+(j-1-M) \tau)$ $(t \in[0, \tau], j=1, \ldots, M)$.

Теорема 1. Если $\varphi \in C_{\mathbb{C}}$ - решение уравнения (4), то вектор-фуункиия $U:[0, \tau] \rightarrow \mathbb{C}^{N}$, заданная формулой $(5)$, является решением задачи (6)-(8). Обратно, если $U:[0, \tau] \rightarrow \mathbb{C}^{N}$ - решение задачи $(6)-(8)$, то бункиия $\varphi \in C_{\mathbb{C}}$, заданная формулой

$$
\begin{gathered}
\varphi(t)=\lambda^{-(m-l)} u_{i}(t-(i-1-(m-l) N) \tau)-\sum_{s=1}^{m-l} \lambda^{-s} \psi(t+(s-1) N \tau) \\
(t \in[(i-1-(m-l) N) \tau,(i-(m-l) N) \tau] ; i=m N-M+1, \ldots, N, \\
\quad \text { если } l=0, \text { и } l=1, \ldots, N, \text { если } 1 \leqslant l \leqslant m-1 ; l=0, \ldots, m-1),
\end{gathered}
$$

является решением уравнения (4).

4. Рассмотрим однородную систему обыкновенных дифференциальных уравнений $(6),(7)$, т. е. $\psi_{i}(t) \equiv 0(t \in[0, \tau], i=1, \ldots, M)$. Обозначим через $S_{\lambda}:[0, \tau] \rightarrow$ $\mathbb{C}^{N \times N}$ фундаментальную матрицу этой системы, такую, что $S_{\lambda}(0)=E$, где 
$\lambda \in \mathbb{C} \backslash\{0\}$. Пусть $e_{\lambda j}(t)$ обозначает $j$-ю строку матрицы $S_{\lambda}(t)$. Введем матрицу $Q(\lambda) \in \mathbb{C}^{N \times N}$ по формуле

$$
Q(\lambda)=\left(\begin{array}{c}
e_{\lambda 1}(0)-\lambda^{-1} e_{\lambda N}(\tau) \\
e_{\lambda 2}(0)-e_{\lambda 1}(\tau) \\
\vdots \\
e_{\lambda N}(0)-e_{\lambda, N-1}(\tau)
\end{array}\right)
$$

и рассмотрим аналитическую функцию $q: \mathbb{C} \backslash\{0\} \ni \lambda \mapsto \operatorname{det} Q(\lambda)$.

Пусть $j_{q}(\lambda)$ обозначает порядок нуля $\lambda \neq 0$ функции $q$. Если $q(\lambda) \neq 0$ и $\lambda \in \mathbb{C} \backslash\{0\}$, положим $j_{q}(\lambda)=0$. Обозначим через $m(\lambda)=m(V, \lambda)$ алгебраическую кратность собственного значения $\lambda \neq 0$. Если $\lambda \in \mathbb{C} \backslash\{0\}$ не является собственным значением оператора $V$, мы положим $m(\lambda)=0$. Обозначим через $j_{1}, \ldots, j_{N_{0}}, N_{0} \leqslant N-1, j_{i}<j_{i+1}$, все индексы, такие, что $a_{j_{\nu}}(\tau) \neq 0$ $\left(\nu=1, \ldots, N_{0}\right)$.

Обозначим через $\widehat{Q}(\lambda) \in \mathbb{C}^{N \times N}$ присоединенную матрицу для $Q(\lambda)$ с элементами $(-1)^{i+j} Q_{j i}(\lambda)$ в $i$-й строке и $j$-м столбце, где $Q_{j i}(\lambda)$ - определитель матрицы, полученной из $Q(\lambda)$ вычеркиванием $j$-й строки и $i$-го столбца. Пусть $\widehat{Q}^{0}(\lambda) \in \mathbb{C}^{N \times\left(N_{0}+1\right)}$ обозначает матрицу с элементами $\widehat{Q}_{\eta, \nu+1}^{0}(\lambda)(\eta=1, \ldots, N$, $\left.\nu=0, \ldots, N_{0}\right)$, заданными формулой

$$
\widehat{Q}_{\eta, \nu+1}^{0}(\lambda)=(-1)^{\eta+j_{\nu}+1} Q_{j_{\nu}+1, \eta}(\lambda) \quad\left(\eta=1, \ldots, N, \nu=0, \ldots, N_{0}\right) .
$$

Положим $\mathscr{N}_{q}=\{\lambda \in \mathbb{C} \backslash\{0\}: q(\lambda)=0\}$.

Теорема 2. Имеют место соотношения $\sigma(V) \backslash\{0\}=\mathscr{N}_{q}$ u $m(V, \lambda) \leqslant$ $j_{q}(\lambda)(N-\operatorname{rank} Q(\lambda))$ для всех $\lambda \in \mathbb{C} \backslash\{0\}$. Если $\kappa$ тому же $\left\|\widehat{Q}^{0}\left(\lambda_{0}\right)\right\| \neq 0$ для некоторого $\lambda_{0} \in \mathscr{N}_{q}$, mо $m\left(V, \lambda_{0}\right)=j_{q}\left(\lambda_{0}\right) u \operatorname{dim} \operatorname{Ker}\left(V-\lambda_{0} I\right)=1$.

Положим $\Gamma_{1}=\{\lambda \in \mathbb{C}:|\lambda|=1\}$.

Из теоремы 2 мы получаем следующее утверждение.

Tеорема 3. Если $\Gamma_{1} \cap \mathscr{N}_{q}=\{-1\}$, rank $Q(-1)=N-1 u q^{\prime}(-1) \neq 0$, mo $2 r$-neриодическое решение $x$ уравнения (1) является гиперболическим. Обратно, если $2 r$-периодическое решение $x$ уравнения (1) является гиперболическим и к тому же $\left\|\widehat{Q}^{0}(-1)\right\| \neq 0$, то $\Gamma_{1} \cap \mathscr{N}_{q}=\{-1\}, \operatorname{rank} Q(-1)=N-1$ u $q^{\prime}(-1) \neq 0$.

5. Покажем теперь, как вычислить матрицу $S_{\lambda}(t)$ в некоторых частных случаях. Предположим, что $a_{i_{1}} \equiv 0(t \in[0, \tau])$ для некоторого $i_{1} \in\{1, \ldots, N\}$. Это условие выполняется, если существует $a>0$, такое, что (I) $f^{\prime}(\xi)=0(|\xi| \geqslant a)$ и (II) $|x(t)| \geqslant a\left(t \in\left[\left(i_{1}-1-M\right) \tau,\left(i_{1}-M\right) \tau\right]\right)$. Тогда, аналогично [1, §4], можно показать, что существует перестановка $p$ индексов $1, \ldots, N$, такая, что для каждого $\lambda \in \mathbb{C} \backslash\{0\}$ соответствующая перестановка строк и столбцов матрицы $S_{\lambda}(t)$ приводит к треугольной матрице $\widetilde{S}_{\lambda}(t)$ с нулями над главной диагональю, функциями $e^{-\mu t}$ на главной диагонали и функциями вида

$e^{-\mu t} \int_{0}^{t} b_{p(j)}\left(\xi_{j}, \lambda\right) \int_{0}^{\xi_{j}} b_{p(j-1)}\left(\xi_{j-1}, \lambda\right) \ldots \int_{0}^{\xi_{k+2}} b_{p(k+1)}\left(\xi_{k+1}, \lambda\right) d \xi_{k+1} \ldots d \xi_{j-1} d \xi_{j}$, 
стоящими в $j$-й строке и $k$-м столбце $(k<j)$, где

$$
b_{p(j)}\left(\xi_{j}, \lambda\right)= \begin{cases}\lambda^{-m} a_{p(j)}\left(\xi_{j}\right), & \text { если } 1 \leqslant p(j) \leqslant n, \\ \lambda^{-(m-1)} a_{p(j)}\left(\xi_{j}\right), & \text { если } n+1 \leqslant p(j) \leqslant N .\end{cases}
$$

Матрица $Q_{\lambda}$ пределяется по формуле (10). Таким образом, в данном случае критерий гиперболичности легко проверяется.

\title{
ЛитерАТУРА
}

1. Вальтер Х.-О., Скубачевский А. Л. Труды ММО, 64, 3-53 (2003). 2. Хейл Дж. Теория функционально-дифференциальных уравнений. Мир, М., 1984. 3. Chow $S$. N., Diekmann O., Mallet-Paret J. Japan J. Appl. Math., 2, No. 2, 433-469 (1985). 4. Chow S. N., Walther H. O. Trans. Amer. Math. Soc., 307, No. 1, 127-142 (1988). 5. Walther H. O. Mem. Amer. Math. Soc., 402 (1989). 6. Diekmann O., van Gils S., Verduyn Lunel S., Walther H. O. Delay Equations. Functional, Complex, and Nonlinear Analysis. SpringerVerlag, New York, 1995. 7. Mallet-Paret J., Sell G. J. Differential Equations, 125, No. 2, 385-440 (1996).

Mathematisches Institut,

Поступило в редакцию Universitaet Giessen, Germany 28 июня 2004 г.

e-mail: Hans-Otto.Walther@math.uni-giessen.de

Московский авиационный институт

e-mail: skub@lector.ru

УДК 517.948

\section{Об аппроксимации решений операторных уравнений методом наименьших квадратов*}

\author{
(C) 2005. М. Л. ГОРБАЧУК
}

Пусть $\mathfrak{H}$ - сепарабельное гильбертово пространство над полем $\mathbb{C}$ комплексных чисел, $(\cdot, \cdot)$ и $\|\cdot\|$ - скалярное произведение и норма в нем, а $A-$ обратимый замкнутый линейный оператор с $\mathscr{D}\left(A^{-1}\right)=\mathfrak{H}(\mathscr{D}(\cdot)$ - область определения оператора).

Рассмотрим уравнение

$$
A u=f, \quad f \in \mathfrak{H},
$$

и будем искать его приближенное решение $u_{n}$ методом наименьших квадратов, т. е. в виде

$$
u_{n}=\sum_{k=1}^{n} \alpha_{k} e_{k}
$$

где $\left\{e_{k}\right\}_{k \in \mathbb{N}}$ - наперед выбранная линейно независимая система векторов из $\mathscr{D}(A)$ (так называемая координатная система), а $\alpha_{k} \in \mathbb{C}$ таковы, что величина

*Работа поддержана Совместным грантом CRDF и правительства Украины UM1-2567OD03. 